\title{
The Gravitational Radiation Emitted by a System Consisting of a Point Particle in Close Orbit around a Schwarzschild Black Hole
}

\author{
Amos S. Kubeka \\ Department of Mathematical Sciences, University of South Africa, Pretoria, South Africa \\ Email: kubekas@unisa.ac.za
}

Received August 5, 2012; revised September 14, 2012; accepted September 20, 2012

\begin{abstract}
We analytically model a relativistic problem consisting of a point-particle with mass $m$ in close orbit around a stationary Schwarzschild black hole with mass $M=1$ using the null-cone formalism when $l=2$. We use the $\delta$-function to model the matter density of the particle. To model the whole problem, we apply the second order differential equation obtained elsewhere for a dynamic thin matter shell around a Schwarzschild black hole. The only thing that changes on the equation is the quasi-normal mode parameter which now represent the orbital frequency of the particle. We compare our results with that of the standard 5.5 PN formalism and found that there is a direct proportionality factor that relates the two results, i.e. the two formalisms.
\end{abstract}

Keywords: Null Formalism; Gravitational Radiation; Schwarzschild Black Hole; PN Formalism; Delta Function; Point Particle; Bondi Mass

\section{Introduction}

To date, numerically speaking black hole systems have been studied only theoretically and this means that all areas of mathematics (both pure and applied) and the computational sciences are heavily utilized in this field. As a result at the moment the research into the formation and the evolution of compact binaries i.e. white dwarfwhite dwarf, neutron star-neutron star, black hole-black hole and colliding black holes is progressing very rapidly and important results are being published [1]. The research into a black hole-neutron star binary system in quasi-equilibrium or in full dynamic motion in either Newtonian (see [2-6] for quasi-equilibrium and [7-13] for dynamic motion) or relativistic theory (see [14-19] for quasi-equilibrium and [20-25] for dynamic motion) is as challenging as that of a black hole-black hole binary system or two colliding black holes. Relativistically speaking, neutron stars binaries and black hole binaries are thought to be the primary sources of the gravitational radiation to be hopefully detected by the ground-based LIGO [26] and for white dwarf binaries by the spacebased LISA [27]. In this paper we analytically study in the Bondi-frame, a binary system consisting of a pointparticle in quasi-orbit around a stationary Schwarzschild black hole. Our main aim shall be to determine the emitted gravitational radiation by the system at $\mathcal{I}^{+}$. The null-cone formalism have also been used numerically to study quite extensively other systems consisting of black hole binaries [28]. The PN formalism has should to be accurate for modeling gravitating systems at the Newtonian regime. So, by comparing the results from these two formalisms for the same physical problem is vitally important in validating our final results. This paper is structured as follows: Section 2 gives the background material. Section 3 define the physical problem to be studied. Section 4 calculates the emitted gravitational radiation at $\mathcal{I}^{+}$.

\section{Background Material}

\subsection{The Null-Cone Formalism}

The Bondi-Sachs formalism uses coordinates $x^{i}=\left(u, r, x^{A}\right)$ based upon a family of outgoing null hypersurfaces. We label these hypersurfaces by $u=$ const., null rays by $x^{A}\left(A=2,3, x^{2}=\theta, x^{3}=\phi\right)$, and the surface area coordinate by $r$. In this coordinates system the Bondi-Sachs metric $[29,30]$ takes the form

$$
\begin{aligned}
\mathrm{d} s^{2}= & -\left[\mathrm{e}^{2 \beta}\left(1+\frac{W}{r}\right)-r^{2} h_{A B} U^{A} U^{B}\right] \mathrm{d} u^{2}-2 \mathrm{e}^{2 \beta} \mathrm{d} u \mathrm{~d} r \\
& -2 r^{2} h_{A B} U^{B} \mathrm{~d} u \mathrm{~d} x^{A}+r^{2} h_{A B} \mathrm{~d} x^{A} \mathrm{~d} x^{B},
\end{aligned}
$$

where $h^{A B} h_{B C}=\delta_{B}^{A}$ and $\operatorname{det}\left(h_{A B}\right)=\operatorname{det}\left(q_{A B}\right)$, with $q_{A B}$ being a unit sphere metric, $U$ is the spin-weighted 
field given by $U=U^{A} q_{A}$. For a Schwarzschild spacetime, $W=-2 M$. We define the complex quantity $J$ by

$$
J=q^{A} q^{B} h_{A B} / 2 .
$$

For the Schwarzschild space-time, we have $J$ and $U$ being zero and thus they can be regarded as a measure of the deviation from spherical symmetry, and in addition, they contain all the dynamic content of the gravitational field in the linearized regime [31]. Usually we can describe this space-time by $\beta=0$ and $W=-2 M$, or by $\beta=\beta_{c}$ (constant) and $W=\left(e^{2 \beta_{c}}-1\right) r-2 M$.

For spherical harmonics we use ${ }_{s} Z_{l m}$ rather than ${ }_{s} Y_{l m}$ as basis functions as follows [32]

$$
\begin{aligned}
{ }_{s} Z_{l m} & =\frac{1}{\sqrt{2}}\left[{ }_{s} Y_{l m}+(-1)^{m}{ }_{s} Y_{l-m}\right] \text { for } m>0 \\
{ }_{s} Z_{l m} & =\frac{i}{\sqrt{2}}\left[(-1)^{m}{ }_{s} Y_{l m}-{ }_{s} Y_{l-m}\right] \text { for } m>0 \\
{ }_{s} Z_{l 0} & ={ }_{s} Y_{l 0},
\end{aligned}
$$

The $s=0$ will be omitted in the case $s=0$, i.e. $Z_{l m}={ }_{0} Z_{l m}$. The ${ }_{s} Z_{l m}$ are orthonormal and real. We assume the following ansatz

$$
\begin{aligned}
& J=\operatorname{Re}\left(J_{0}(r) \mathrm{e}^{\mathrm{i} \sigma u}\right) \partial^{2} Z_{l m}, \\
& U=\operatorname{Re}\left(U_{0}(r) \mathrm{e}^{\mathrm{i} \sigma u}\right) \partial Z_{l m}, \\
& \beta=\operatorname{Re}\left(\beta_{0}(r) \mathrm{e}^{\mathrm{i} \sigma u}\right) Z_{l m}, \\
& \omega=\operatorname{Re}\left(\omega_{0}(r) \mathrm{e}^{\mathrm{i} \sigma u}\right) Z_{l m},
\end{aligned}
$$

where $r_{0}$ is the position of the matter shell, and $\sigma$ the complex frequency mode which is physical damped and which further means that $\operatorname{Im}(\sigma)>0$. In the Bondi frame, the field equations splits into;

- the hypersurface equations and the evolution equations given by

$$
\begin{gathered}
R_{r r}: \frac{4}{r} \beta_{, r}=8 \pi T_{r r} \\
q^{A} R_{r A}: \frac{1}{2 r}\left(4 ð \beta-2 r ð \beta_{, r}+r \bar{\partial} J_{, r}+r^{3} U_{, r r}+4 r^{2} U_{, r}\right) \\
=8 \pi q^{A} T_{r A} \\
h^{A B} R_{A B}:(4-2 ð \bar{\partial}) \beta+\frac{1}{2}\left(\bar{\partial}^{2} J+\check{\partial}^{2} \bar{J}\right) \\
+\frac{1}{2 r^{2}}\left(r^{4} \bar{\partial} \bar{U}+r^{4} \bar{\partial} U\right)_{, r}-2 \omega_{, r}=8 \pi\left(h^{A B} T_{A B}-r^{2} T\right) \\
q^{A} q^{B} R_{A B}:-2 \partial^{2} \beta+\left(r^{2} \partial U\right)_{, r}-2(r-M) J_{, r} \\
-\left(1-\frac{2 M}{r}\right) r^{2} J_{, r r}+2 r(r J)_{, u r}=8 \pi q^{A} q^{B} T_{A B},
\end{gathered}
$$

- and the constraint equations for off the matter shell in the case of vacuum given by

$$
\begin{aligned}
& R_{u u}: \frac{1}{2 r^{3}}(r(r-2 M)) \omega_{, r r}+ð \bar{\partial} \omega+2(r-2 M) ð \bar{\varnothing} \beta \\
& -M r(\widetilde{\partial U}+\bar{\partial} U)-r^{3}(ð \bar{U}+\bar{\partial} U)_{, u}+2 r \omega_{, u}=0, \\
& R_{u r}: \frac{1}{4 r^{2}}\left(2 r \omega_{, r r}+4 ð \bar{\delta} \beta-\left(r^{2} \check{\partial} \bar{U}+r^{2} \bar{\partial} U\right)_{, r}\right)=0, \\
& q^{A} R_{u A}: \frac{1}{4 r^{2}}\left(2 r \partial \omega_{, r}-2 \partial \omega+2 r^{2}(r-2 M)\left(4 U_{, r}+r U_{, r r}\right)\right. \\
& \left.+4 r^{2} U+r^{2}\left(ð \bar{\partial} U-\check{\partial}^{2} \bar{U}\right)+2 r^{2} \bar{\partial} J_{, u}-2 r^{4} U_{, u r}-4 r^{2} \check{\partial} \beta_{, u}\right) \\
& =0 \text {, }
\end{aligned}
$$

Ref. [32] got the following second order differential equation when solving the above systems of ordinary differential equations for the Schwarzschild background;

$$
\begin{aligned}
& x^{3}(1-2 x M) \frac{\mathrm{d}^{2} J_{2}}{\mathrm{~d} x^{2}}+2 \frac{\mathrm{d} J_{2}}{\mathrm{~d} x}\left(2 x^{2}+\mathrm{i} \sigma x-7 x^{3} M\right) \\
& -2\left(x\left(l^{2}+l-2\right) / 2+8 M x^{2}+\mathrm{i} \sigma\right) J_{2}=0
\end{aligned}
$$

where $J_{2}(x) \equiv \mathrm{d}^{2} J_{0+} / \mathrm{d} x^{2}$ and $x=1 / r, x$ is the compactification factor in this language. Bishop et al. [33] solved Equation (12) numerically and obtained interesting quasi-normal modes results of a Schwarzschild white hole. However in this paper, we are going to solved it for a different problem since we can apply the same physical settings in the Bondi-frame to model our problem with $\sigma$ having a different physical meaning as we shall see later.

\subsection{An Analytic Algorithm for Calculating the Gravitational News}

We shall use the following algorithm to calculate the gravitational radiation from the system.

- First we use Equation (12) and the constraints Equations (9)-(11) to get the junction conditions for the Bondi-Sachs matric variables $U, \omega$ and $J$ at the boundary i.e. shell,

- Second we test if $J, J_{, r}, U, U_{, r}$, and $\omega$ are smooth across the boundary and if this is true, we then

- Calculate the News function at $\mathcal{I}^{+}$.

\section{The Physical Problem}

We consider a system consisting of a point-particle with mass $\mathrm{m}$ in quasi-orbit around a stationary Schwarzschild black hole at $r_{0}$ with mass $M$ for $\ell$ is 2 . We simplify the coordinate dynamics of the center of mass of the system by doing a mathematical trick. That is, we place a second point-particle directly opposite the first pointparticle at $r_{0}$. That means the center of mass will remain at the origin i.e. at the black hole during the duration of the orbit. The total distance between the point-particles is 
$2 r_{0}$. This trick has the consequence that the emitted gravitational radiation will be amplified by a factor of two which in the final analysis we divide the final result by two. This is as a result of the introduced point-particle. This procedure is physical correct as long as the pointparticles are equal and in equidistance in a quasi-circular orbit. We take the initial position of the first particle to be at $\pi / 2$ and $v u$ for the $\theta$ and $\phi$ respectively. We also take the initial position of the second particle to be at $\pi / 2$ and $v u+\pi$ for $\theta$ and $\phi$ respectively. We define $v$ as the orbital frequency and $u$ as the orbital period of the particles.

The dynamics of this problem is governed by Equation (12) and for our numerical calculation purposes we shall use its Ricatti form [33]

$$
\frac{\mathrm{d} v}{\mathrm{~d} x}=1+\frac{2 v}{x^{2}(1-2 x)}\left((x-v)\left(2+\frac{i v}{x}\right)-x(7 x+8 v)\right)
$$

where $v$ is the orbital period of the system.

\section{The Emitted Gravitational Radiation}

\subsection{The Linear Expansion of the Light Rays}

We start by applying Equation (5) with $T_{r r}$ given by

$$
\rho\left(1-\frac{2 M}{r}\right)^{-1}
$$

where the matter density $\rho$ in the background spacetime is given by

$$
\rho=\frac{m}{r_{0}^{2}} \delta\left(r-r_{0}\right)\left(\theta-\frac{\pi}{2}\right)[\delta(\phi-v u)+\delta(\phi-v u-\pi)] .
$$

Inside the orbital radius $r<r_{0}$ we set

$$
\beta=0,
$$

and outside the orbital radius $r>r_{0}$ we set

$$
\beta=\sum_{l m} \beta_{l m} Z_{l m} .
$$

Now integrating with respect to $r$ we get

$$
\int \beta_{, r} \mathrm{~d} r=\int 2 \pi r \rho\left(1-\frac{2 M}{r}\right)^{-1} \mathrm{~d} r
$$

i.e.

$$
\begin{aligned}
& \sum_{l m} \beta_{l m} Z_{l m} \\
& =\frac{2 \pi m}{r_{0}}\left(1-\frac{2 M}{r}\right)^{-1} \delta\left(\theta-\frac{\pi}{2}\right)[\delta(\phi-v u)+\delta(\phi-v u-\pi)]
\end{aligned}
$$

By multiplying Equation (19) with $Z_{l^{\prime} m^{\prime}}$ we get

$$
\begin{aligned}
Z_{l^{\prime} m^{\prime}} \sum_{l m} \beta_{l m} Z_{l m}= & \frac{2 \pi m}{r_{0}}\left(1-\frac{2 M}{r}\right)^{-1} \delta\left(\theta-\frac{\pi}{2}\right) \\
& \cdot\left[Z_{l^{\prime} m^{\prime}} \delta(\phi-v u)+Z_{l^{\prime} m^{\prime}} \delta(\phi-v u-\pi)\right]
\end{aligned}
$$

and integrating over the sphere it simplifies to

$$
\begin{aligned}
\beta_{l^{\prime} m^{\prime}}= & \frac{2 \pi m}{r_{0}}\left(1-\frac{2 M}{r}\right)^{-1} \\
& \cdot\left[Z_{l^{\prime} m^{\prime}}\left(\frac{\pi}{2}, v u\right)+Z_{l^{\prime} m^{\prime}}\left(\frac{\pi}{2}, v u+\pi\right)\right] .
\end{aligned}
$$

From Equation (21), for $m^{\prime} \neq 0$ we the gravitational radiation otherwise we don't, and that $\beta_{l^{\prime} m^{\prime}}$ are generally non-zero for even $l$ and $m^{\prime}$. We now consider the case $l^{\prime}=2$ and we note that

$$
\begin{gathered}
\beta_{21}=0, \\
\beta_{2,-1}=0,
\end{gathered}
$$

and that

$$
\beta_{20} \neq 0 .
$$

The problem with $\beta_{20}$ is that it does not vary in time so this mode does not contain the gravitational radiation. So we are more interested in $\beta_{22}$ and $\beta_{2,-2}$ modes. Using the following normalized spherical harmonics

$$
\begin{aligned}
Y_{22} & =\frac{1}{4} \sqrt{\frac{15}{2 \pi}} \sin ^{2} \theta \mathrm{e}^{2 i \phi}, \\
Y_{2,-2} & =\frac{1}{4} \sqrt{\frac{15}{2 \pi}} \sin ^{2} \theta \mathrm{e}^{-2 i \phi},
\end{aligned}
$$

and the fact that

$$
\begin{aligned}
Z_{22} & =\frac{1}{\sqrt{2}}\left(Y_{22}+Y_{2,-2}\right), \\
Z_{2,-2} & =\frac{\mathrm{i}}{\sqrt{2}}\left(Y_{2,-2}-Y_{22}\right),
\end{aligned}
$$

we get

$$
Z_{22}=\frac{\sqrt{2}}{4} \sqrt{\frac{15}{2 \pi}} \sin ^{2} \theta \cos 2 \phi
$$

and

$$
Z_{2,-2}=\frac{\sqrt{2}}{4} \sqrt{\frac{15}{2 \pi}} \sin ^{2} \theta \sin 2 \phi .
$$

Thus from Equation (21)

$$
\begin{aligned}
\beta_{22} & =\frac{2 \pi m}{r_{0}}\left(1-\frac{2 M}{r_{0}}\right)^{-1} \frac{\sqrt{2}}{4} \sqrt{\frac{15}{2 \pi}} \cos (2 v u) 2, \\
& =\frac{m}{r_{0}}\left(1-\frac{2 M}{r_{0}}\right)^{-1} \sqrt{15 \pi} \cos (2 v u),
\end{aligned}
$$


and similarly

$$
\beta_{2,-2}=\frac{m}{r_{0}}\left(1-\frac{2 M}{r_{0}}\right)^{-1} \sqrt{15 \pi} \sin (2 v u)
$$

and then finally we write

$$
\begin{aligned}
\beta= & \frac{m}{r_{0}} \sqrt{15 \pi}\left(1-\frac{2 M}{r_{0}}\right)^{-1} \\
& \cdot\left(\operatorname{Re}\left\{\mathrm{e}^{2 \mathrm{i} v u}\right\} Z_{22}+\operatorname{Re}\left\{-\mathrm{ie}^{2 \mathrm{i} v u}\right\} Z_{2,-2}\right)
\end{aligned}
$$

Now taking $M=1$, Equation (35) then becomes

$$
\begin{aligned}
\beta= & \frac{m}{r_{0}} \sqrt{15 \pi}\left(1-\frac{2}{r_{0}}\right)^{-1} \\
& \cdot\left(\operatorname{Re}\left\{\mathrm{e}^{2 \mathrm{i} v u}\right\} Z_{22}+\operatorname{Re}\left\{-\mathrm{ie}^{2 \mathrm{i} v u}\right\} Z_{2,-2}\right)
\end{aligned}
$$

Finally, we divide Equation (34) by a factor of 2 to appropriate it for a single point-particle in orbit around a Schwarzschild black hole. We are able to do this because for $\theta=\pi / 2=0$ we have $Z_{2,1}=0$. Therefore from here onwards, the calculations will be that of a single pointparticle. Equation (34) now becomes

$$
\begin{aligned}
\beta= & \frac{1}{2} \frac{m}{r_{0}} \sqrt{15 \pi}\left(1-\frac{2}{r_{0}}\right)^{-1} \\
& \cdot\left(\operatorname{Re}\left\{\mathrm{e}^{2 \mathrm{i} v u}\right\} Z_{22}+\operatorname{Re}\left\{-\mathrm{ie}^{2 \mathrm{i} v u}\right\} Z_{2,-2}\right)
\end{aligned}
$$

\subsection{The Gravitational Radiation}

We assume that the orbit is at the innermost stable circular orbit (ISCO), so that $r=r_{0}=6$. We then found the change in the Schwarzschild coordinate time $\mathcal{I}$ for one complete revolution of 92.3436 from which we found the orbital frequency $v$ of 0.0680 .

To now find the numerical solutions to continue Equation (13) we make the spatial coordinate transformation of $x=1 / r$ which then imply that the ISCO is now at $x_{m n}=1 / 6$. The numerical computations are done in the domains

$$
D_{+}=\left\{0<x<x_{m n}\right\} \text { and } D_{-}=\left\{x_{m n}<x<0.5\right\},
$$

with numerical solutions $v_{+}(x)$ and $v_{-}(x)$ respectively. We start the calculation with the transformed Equation (12) given by

$$
\begin{aligned}
U(x)= & 2 \beta_{0} x-\frac{1}{2} x^{4}(1-2 x M) \frac{\mathrm{d}^{3}}{\mathrm{~d} x^{3}} J(x) \\
& -x^{3}\left(x-2 x^{2} M+\mathrm{i} v\right) \frac{\mathrm{d}^{2}}{\mathrm{~d} x^{2}} J(x) \\
& +x\left(2 x+2 x^{2} M+\mathrm{i} v\right) \frac{\mathrm{d}}{\mathrm{d} x} J(x)-\mathrm{i} v J(x)
\end{aligned}
$$

where $U_{+}(x), U_{-}(x)$ are the Bondi metric functions, and $\beta_{0+}, \beta_{0-}$ are the values of the expansion of the light rays $\beta$ given by Equation (35) in the exterior and interior domains respectively. N.B the derivatives of $J$ should not be worked out numerically, but should be worked out analytically in terms of $J_{1}, J_{2}$ and $v$ from Equation (13) to be found with $v=0.0680$.

We define the general solutions for $J_{2}(x)$ at $x_{m n}$ outside and inside the orbital radius respectively as

$$
\begin{aligned}
& J_{+}(x)=c 4+c 1 x+c 2 J_{0+}(x), \\
& J_{-}(x)=c 9+c 6 x+c 7 J_{0-}(x),
\end{aligned}
$$

where $c 4, c 1, c 2, c 9, c 6$ and $c 7$ are constants to be determined numerically. The functions $J_{0+}(x)$ and $J_{0-}(x)$ are analytic near $x_{m n}$ and therefore can be Taylor expand as

$$
\begin{aligned}
J_{+}(x)= & J_{0+}\left(x_{m n}\right)+\left(x-x_{m n}\right) \frac{\mathrm{d}}{\mathrm{d} x} J_{0+}(x) \\
& +\frac{\left(x-x_{m n}\right)^{2}}{2} \frac{\mathrm{d}^{2}}{\mathrm{~d} x} J_{0+}(x)+\frac{\left(x-x_{m n}\right)^{3}}{6} \frac{\mathrm{d}^{3}}{\mathrm{~d} x} J_{0+}(x), \\
J_{-}(x)= & J_{0-}\left(x_{m n}\right)+\left(x-x_{m n}\right) \frac{\mathrm{d}}{\mathrm{d} x} J_{0-}(x) \\
& +\frac{\left(x-x_{m n}\right)^{2}}{2} \frac{\mathrm{d}^{2}}{\mathrm{~d} x} J_{0-}(x)+\frac{\left(x-x_{m n}\right)^{3}}{6} \frac{\mathrm{d}^{3}}{\mathrm{~d} x} J_{0-}(x),
\end{aligned}
$$

which then results in Equations (38) and (41) being analytic near $x_{m n}$. We used Matlab ode45 solver to find numerical solutions of the above derivatives in Equations (40) and (41). We used stringent numerical conditions to get the results to about seven significant figures with RelTol of $10^{-12}$, AbsTol of $10^{-12}$, and the MaxStep of $2 \times 10^{-6}$ and the results we found to be

$$
\begin{aligned}
& \frac{\mathrm{d}}{\mathrm{d} x} J_{0+}(x)=29144-2.280672 \times 10^{5} \mathrm{i}, \\
& \frac{\mathrm{d}^{2}}{\mathrm{~d} x} J_{0+}(x)=2.865551 \times 10^{6}-1.52335130 \times 10^{7} \mathrm{i}, \\
& \frac{\mathrm{d}^{3}}{\mathrm{~d} x} J_{0+}(x)=4.8870 \times 10^{7}-1.8591431 \times 10^{9} \mathrm{i},
\end{aligned}
$$

and

$$
\begin{aligned}
& \frac{\mathrm{d}}{\mathrm{d} x} J_{0-}(x)=13.04337-1.31529 \mathrm{i} \\
& \frac{\mathrm{d}^{2}}{\mathrm{~d} x} J_{0-}(x)=1.54689 \times 10^{2}-3.19980 \times 10^{1} \mathrm{i}, \\
& \frac{\mathrm{d}^{3}}{\mathrm{~d} x} J_{0-}(x)=-1.12428 \times 10^{3}-1.25311 \times 10^{3} \mathrm{i} .
\end{aligned}
$$

We have tested for the consistency of the above results by using other Matlab solvers; ode23 and ode15s (which 
uses the Gears method i.e. backward differentiation formulas) and also observed the accuracy of about 15 significant figures. We went further with the test using ode23t which uses the trapezoidal rule, ode $23 \mathrm{~s}$ which is a modified Rosenbrock formula of order 2, and ode23tb which is an implicit Runge Kutta as opposed to ode45 and ode23 and found the consistency of about 8 significant figures and as opposed to 15 significant figures which is also accurate enough. This illustrate how accurate and valid the results are. These results are very crucial in obtaining the emitted gravitational radiation and hence determining the extent of their consistency is of the most paramount importance to obtaining accurate final results.

From the hypersurface equation Equation (7) rewritten as

$$
-2 x^{2} \omega_{, x}=2\left(2-L_{2}\right) \beta_{0}+L_{2}\left(L_{2}+2\right) J-x^{4}\left(x^{-4} L_{2} U\right)_{, x}
$$

we are able to the Bondi metric function $\omega_{+}(r)$ and $\omega_{-}(r)$. But to find the solution the integration should be done analytically where possible. We only need a solution which is valid in a neighborhood of $x=x_{0}$. Henceforth, it is convenient to make the coordinate transformation $x \rightarrow r=1 / x$. Equation (48) can further be rewritten as

$$
2\left(2-L_{2}\right) \beta_{0}+L_{2}\left(L_{2}+2\right) J+\frac{1}{r^{2}}\left(r^{4} L_{2} U\right)_{, r}=2 \omega_{, r},
$$

where for $l=2$ we have $L_{2}=-6$. The constraints equations Equations (9), (10), and (11) now simplifies to

$$
\begin{aligned}
& R_{u u}: \frac{1}{2 r^{3}}\left(\left(r^{2}-2 M r\right) \omega_{, r r}-6 \omega-12(r-2 M) \beta_{0}+12 M r U\right. \\
&-\left.4 r(r-2 M) \mathrm{i} v \beta_{0}+12 r^{3} \mathrm{i} v U+2 r \mathrm{i} v \omega\right)=0, \\
& q^{A} R_{u A}: \frac{1}{2 r^{2}}\left(r \omega_{, r}-\omega+4 r^{3} U_{, r}+r^{4} U_{, r r}+2 r^{2} U-2 M r^{3} U_{, r r}\right. \\
&\left.-8 M r^{2} U_{, r}-r^{2} \mathrm{i} v J-r^{4} \mathrm{i} v U_{, r}-2 r^{2} \mathrm{i} v \beta_{0}\right)=0 .
\end{aligned}
$$

which we then apply in the domains $D_{+}$and $D_{-}$. Since these constraints are not completely analytic, this means that we should only evaluate them at the ISCO. We use them among others to eliminate the constants $c 1$, $c 2, c 6$, and $c 7$. We now assume that we end up with the solutions

$$
\begin{aligned}
& \omega_{+}(x)=c 5+\omega_{0+}(x), \omega_{-}(x)=c 10+\omega_{0-}(x), \\
& \text { with } \omega_{0+}(x)=\omega_{0-}(x)=0 .
\end{aligned}
$$

Thus, from the constraints $R_{u u-}\left(r_{0}\right), R_{u u+}\left(r_{0}\right)$, $R_{u r-}\left(r_{0}\right), R_{u r+}\left(r_{0}\right), q^{A} R_{u A-}\left(r_{0}\right), q^{A} R_{u A+}\left(r_{0}\right)$, and the hypersurface Equation (49), we found the metric variables $U_{+}\left(r_{0}\right), U_{-}\left(r_{0}\right), \omega_{+}\left(r_{0}\right)$, and $\omega_{-}\left(r_{0}\right)$. From which the expressions of the constants $c 9, c 7, c 5$, and $c 10$, were found.

We now impose the Bondi gauge conditions:

$$
\beta_{0+}=0, \quad c 4=0,
$$

which means that for large $r, \beta_{0+}=0$ at $\mathcal{I}^{+}$imply that the coordinate time is the same as proper time and that the regularity at $\mathcal{I}^{+}$require $c 4=0$. We also impose the following junction conditions at $r_{0}$ :

$$
\begin{gathered}
J_{+}\left(r_{0}\right)=J_{-}\left(r_{0}\right), \\
2 \mathrm{~cm} U_{+}\left(r_{0}\right)=U_{-}\left(r_{0}\right), \\
\beta_{0-}=-2 \pi r_{0} \rho\left(1-\frac{2 M}{r_{0}}\right)^{-1} \\
\omega_{+}\left(r_{0}\right)-\omega_{-}\left(r_{0}\right)=-4 \pi r^{2} \rho .
\end{gathered}
$$

From the junction conditions, we were able to find the exact numerical values of the constants $c 1, c 2$, and $c 6$ at $r 0=6$. The exact numerical values of the constants $c 9, c 7, c 5$, and $c 10$ were then found by substituting the values of $c 1, c 2$, and $c 6$ back into their expressions. From here we were then able to plot the graphs of the Bondi metric functions $J_{-}(r 0), J_{+}(r 0)$, $U_{-}(r 0), U_{+}(r 0), \omega_{-}(r 0)$, and $\omega_{+}(r 0)$ as observed in the following graphs;

Physically the metric functions $J$ and $U$ have the smooth asymptotic expansion characteristic through out the entire computational domain and this property is confirmed in Figures 1 and 2. The metric function $\omega$ do

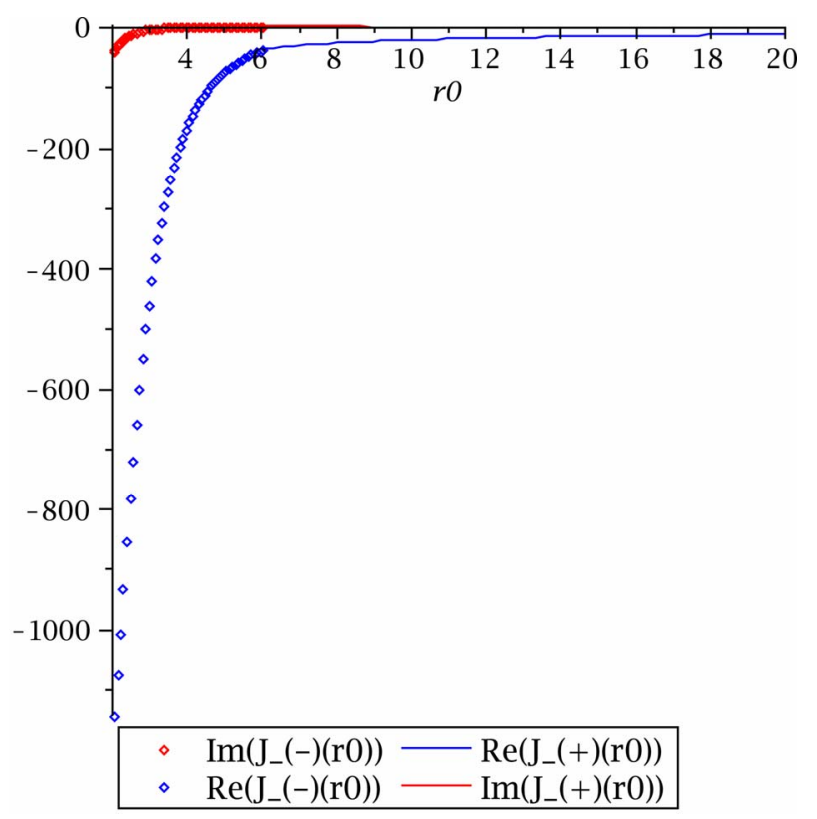

Figure 1. The graph of $\operatorname{Re}\left(J_{-}(r 0)\right), \operatorname{Im}\left(J_{-}(r 0)\right)$ and $\operatorname{Re}\left(J_{+}(r 0)\right)$, $\operatorname{Re}\left(J_{+}(r 0)\right)$ for the Schwarzschild space-time. $v=0.07$ and $\ell$ $=2$. 


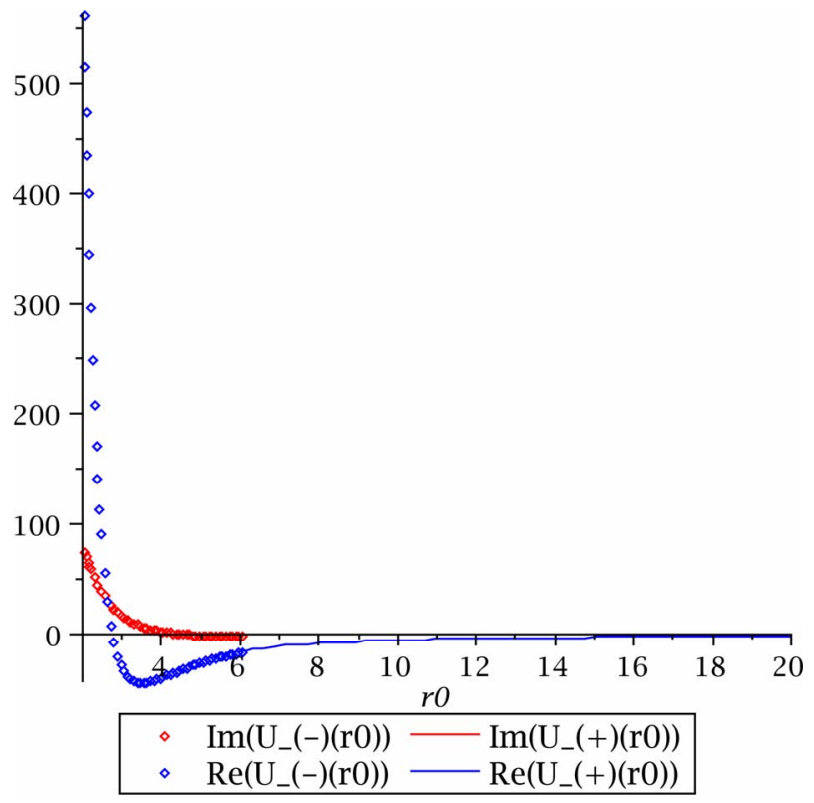

Figure 2. The graph of $\operatorname{Re}\left(U_{-}(r 0)\right), \operatorname{Im}\left(U_{-}(r 0)\right)$ and $\operatorname{Re}\left(U_{+}(r 0)\right)$, $\operatorname{Im}\left(U_{+}(r 0)\right)$ for the Schwarzschild space-time. $v=0.07$ and $\ell=2$.

not have this physical property as can be confirmed in Figure 3 but this function is crucial in the calculation procedure of the gravitation radiation in the entire domain. Physically the function $J$ in the only one that have the time derivative and thus carries the gravitational radiation information to calculated at $\mathcal{I}^{+}$and that all the other Bondi metric functions are intergrated radially from $\Gamma$ to $\mathcal{I}^{+}$. The above results indicate that the junction conditions at $r 0=6$ where implemented correctly and that our numerical methods and the analytical algorithms we implemented to calculating the gravitational radiation worked properly as intended.

Then finally, since we are in the Bondi gauge, we found the gravitational news to be

$$
\mathcal{N}_{+}=\frac{1}{2} \operatorname{Re}(c \operatorname{li} v \exp (\mathrm{i} v u))\left(\sqrt{-(l-1) L_{2}(l+2)}\right)_{2} Z_{l m},
$$

which then further simplify to

$$
\mathcal{N}_{+}=\operatorname{Re}(-0.1889 \mathrm{~m}-0.2975 \mathrm{im}),
$$

with the Bondi mass loss $-0.0028 \mathrm{~m}^{2}$. We compare our results with that of 5.5 PN formalism by Poisson [34] and Sasaki et al. [35] which they found the gravitational radiation of the same system like ours with the same physical conditions as in this paper of about $-0.001 \mu^{2}$. From the analysis is seems clear to us that some how there is a factor of about two or three that propositionally relates the two formalisms in studying the gravitational radiation in the newtonian regime. This is fact that still need to be looked at in the near future.

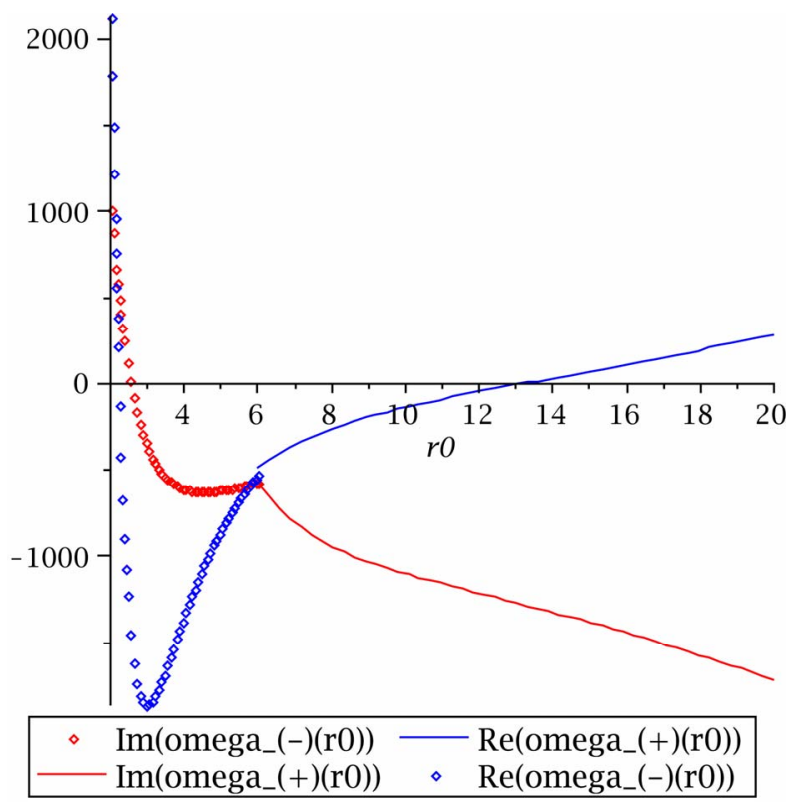

Figure 3. The graph of $\operatorname{Re}\left(\omega_{-}(r 0)\right), \operatorname{Im}\left(\omega_{-}(r 0)\right)$ and $\operatorname{Re}\left(\omega_{+}(r 0)\right)$, $\operatorname{Im}\left(\omega_{+}(r 0)\right)$ for the Schwarzschild space-time. $v=0.07$ and $\ell=2$.

\section{Conclusion}

The work presented here provides us with further future research opportunities to apply the analytic method presented here in the Bondi-frame, to real astrophysics problems involving all sorts of relativistic objects to calculate and analyze the emitted gravitational radiation at null infinity. The next step will be to apply this method to a real relativistic astrophysics problem involving a Kerr background.

\section{Acknowledgements}

I would like to thank Professor Nigel Bishop and Dr. Melusi Khumalo for suggesting improvements of the manuscript. I would also like to thank the National Research Foundation of South Africa under GUN 2053724 for financial support.

\section{REFERENCES}

[1] K. A. Postnov and L. R. Yungelson, "The Evolution of Compact Binaries Star Systems," Living Reviews in Relativity, Vol. 9, 2006, p. 6. http://www.livingreviews.org/lrr-2006-6

[2] S. Chandrasekhar, "Ellipsoidal Figures of Equilibrium," Yale University Press, New Heaven, 1969.

[3] L. G. Fishbone, "The Relativistic Roche Problem. I. Equilibrium Theory for a Body in Equatorial, Circular Orbit around a Kerr Black Hole," Astrophysical Journal, Vol. 185, 1973, pp. 43-68. doi:10.1086/152395

[4] M. Ishii, M. Shibata and Y. Mino, "Black Hole Tidal 
Problem in the Fermi Normal Coordinates," Physical Review D, Vol. 71, No. 4, 2005, Article ID: 044017. doi:10.1103/PhysRevD.71.044017

[5] D. Lai and A. G. Wiseman, "Innermost Stable Circular Orbit of Inspiraling Neutron-Star Binaries: Tidal Effects, Post-Newtonian Effects, and the Neutron-Star Equation of State," Physical Review D, Vol. 54, No. 6, 1996, pp. 3958-3964. doi:10.1103/PhysRevD.54.3958

[6] M. C. Miller, "Prompt Mergers of Neutron Stars with Black Holes," Astrophysical Journal, Vol. 626, No. 1, 2005, p. L41. doi:10.1086/431583

[7] B. Mashhoon, "On Tidal Phenomena in a Strong Gravitational Field," Astrophysical Journal, Vol. 705, 1975, pp. 705-716. doi: $10.1086 / 153560$

[8] B. Carter and J. P. Luminet, "Tidal Compression of a Star by a Large Black Hole," Astronomy \& Astrophysics, Vol. 121, 1983, pp. 97-113.

[9] B. Carter and J. P. Luminet, "Mechanics of the Affine Star Model," Monthly Notices of the Royal Astronomical Society, Vol. 212, 1985, pp. 23-55.

[10] W. H. Lee, "Newtonian Hydrodynamics of the Coalescence of Black Holes with Neutron Stars-III. Irrotational Binaries with a Stiff Equation of State," Monthly Notices of the Royal Astronomical Society, Vol. 318, No. 2, 2000, pp. 606-624. doi:10.1046/j.1365-8711.2000.03870.x

[11] W. H. Lee, "Newtonian Hydrodynamics of the Coalescence of Black Holes with Neutron Stars-IV. Irrotational Binaries with a Soft Equation of State," Monthly Notices of the Royal Astronomical Society, Vol. 328, No. 2, 2001, pp. 583-600. doi:10.1046/j.1365-8711.2001.04898.x

[12] S. Kobayashi, P. Laguna, E. S. Phinney and P. Mészáros, "Gravitational Waves and X-Ray Signals from Stellar Disruption by a Massive Black Hole," Astronomy \& Astrophysics, Vol. 615, No. 2, 2004, p. 855. doi: $10.1086 / 424684$

[13] S. Rosswog, R. Speith and G. A. Wynn, "Accretion Dynamics in Neutron Star-Black Hole Binaries," Monthly Notices of the Royal Astronomical Society, Vol. 351, No. 4, 2004, pp. 1121-1133. doi:10.1111/j.1365-2966.2004.07865.x

[14] T. W. Baumgarte, M. L. Skoge and S. L. Shopiro, "Black Hole-Neutron Star Binaries in General Relativity: Quasiequilibrium Formulation," Physical Review D, Vol. 70, No. 6, 2004, Article ID: 064040. doi:10.1103/PhysRevD.70.064040

[15] P. Grandclément, “Accurate and Realistic Initial Data for Black Hole-Neutron Star Binaries," Physical Review D, Vol. 74, No. 12, 2006, Article ID: 124002. doi:10.1103/PhysRevD.74.124002

[16] P. Grandclément, "Erratum: Accurate and Realistic Initial Data for Black Hole-Neutron Star Binaries," Physical Review D, Vol. 74, 2007, Article ID: 129903(E).

[17] K. Taniguchi, T. W. Baumgarte, J. A. Faber and S. L. Shapiro, "Quasiequilibrium Black Hole-Neutron Star Binaries in General Relativity," Physical Review D, Vol. 75, No. 8, 2007, Article ID: 084005.

\section{doi:10.1103/PhysRevD.75.084005}

[18] K. Taniguchi, T. W. Baumgarte, J. A. Faber and S. L. Shapiro, "Black Hole-Neutron Star Binaries in General Relativity: Effects of Neutron Star Spin," Physical Review D, Vol. 72, No. 4, 2005, Article ID: 044008. doi:10.1103/PhysRevD.72.044008

[19] K. Taniguchi, T. W. Baumgarte, J. A. Faber and S. L. Shapiro, Physical Review D, Vol. 74, 2006, Article ID: 041502(R).

[20] J. A. Faber, T. W. Baumgarte, S. L. Shapiro and K. Taniguchi, "General Relativistic Binary Merger Simulations and Short Gamma-Ray Bursts," Astrophysical Journal, Vol. 641, No. 2, 2006, p. L93. doi:10.1086/504111

[21] J. A. Faber, T. W. Baumgarte, S. L. Shapiro, K. Taniguchi and F. A. Rasio, "Dynamical Evolution of Black Hole-Neutron Star Binaries in General Relativity: Simulations of Tidal Disruption," Physical Review D, Vol. 73, No. 2, 2006, Article ID: 024012. doi:10.1103/PhysRevD.73.024012

[22] F. Löffler, L. Rezzollas and M. Ansorg, "Numerical Evolutions of a Black Hole-Neutron Star System in Full General Relativity: Head-On Collision," Physical Review D, Vol. 74, No. 10, 2006, Article ID: 104018. doi:10.1103/PhysRevD.74.104018

[23] M. Shibata and K. Uryū, "Merger of Black Hole-Neutron Star Binaries: Nonspinning Black Hole Case," Physical Review D, Vol. 74, 2006, Article ID: 121503(R).

[24] M. Shibata and K. Uryū, "Merger of Black Hole-Neutron Star Binaries in Full General Relativity," Classical and Quantum Gravity, Vol. 24, No. 12, 2007, p. S125. doi:10.1088/0264-9381/24/12/S09

[25] C. F. Sopuerta, U. Sperhake and P. Laguna, "Hydrowithout-Hydro Framework for Simulations of Black Hole-Neutron Star Binaries," Classical and Quantum Gravity, Vol. 23, No. 16, 2006, p. S579. doi:10.1088/0264-9381/23/16/S15

[26] B. C. Barish and R. Weiss, "LIGO and the Detection of Gravitational Waves," Physics Today, Vol. 52, No. 10, 1990, p. 44. doi: $10.1063 / 1.882861$

[27] A. Coory, A. J. Farmer and N. Seto, "The Optical Identification of Close White Dwarf Binaries in the Laser Interferometer Space Antenna Era," Astrophysical Journal Letters, Vol. 601, No. 1, 2004, p. L47. doi: $10.1086 / 381780$

[28] L. Lehner, "Gravitational Radiation from Black Hole Spacetime," Ph.D. Thesis, University of Pittsburg, Pittsburg, 1998.

[29] H. Bondi, M. J. G. van der Burg and A. W. K. Metzner, "Gravitational Waves in General Relativity. VII. Waves from Axi-Symmetric Isolated Systems," Proceedings of the Royal Society A, Vol. 269, No. 1336, 1962, pp. 21-52. doi:10.1098/rspa.1962.0161

[30] R. K. Sachs, "Gravitational Waves in General Relativity. VIII. Waves in Asymptotically Flat Space-Time," Proceedings of the Royal Society A, Vol. 270, 1962, pp. 103-126.

[31] N. T. Bishop, R. Gómez, L. Lehner, M. Maharaj and J. 
Winicour, "High-Powered Gravitational News," Physical Review D, Vol. 56, No. 10, 1997, pp. 6298-6309. doi:10.1103/PhysRevD.56.6298

[32] N. T. Bishop, "Linearized Solutions of the Einstein Equations within a Bondi-Sachs Framework, and Implications for Boundary Conditions in Numerical Simulations," Classical and Quantum Gravity, Vol. 22, No. 12, 2005, p. 2393. doi:10.1088/0264-9381/22/12/006

[33] N. T. Bishop and A. S. Kubeka, "Quasinormal Modes of a Schwarzschild White Hole," Physical Review D, Vol.
80, No. 6, 2009, Article ID: 064011. doi:10.1103/PhysRevD.80.064011

[34] E. Poisson, "Gravitational Radiation from a Particle in Circular Orbit around a Black Hole. I. Analytical Results for the Nonrotating Case," Physical Review D, Vol. 47, No. 4, 1993, p. 1497. doi:10.1103/PhysRevD.47.1497

[35] M. Sasaki and H. Tagoshi, “Analytic Black Hole Perturbation approach to Gravitational Radiation," Living Reviews in Relativity, Vol. 6, 2003, p. 6. 


\section{The Constraints Computed at $r_{0}$}

$$
\begin{aligned}
R_{u u-}\left(r_{0}\right)= & 1.000000000 \times 10^{-9}\left(-200 r 0^{9} c 7+6.005358575 \times 10^{19} \mathrm{i} c 7 r 0^{3}+6.8000000 \times 10^{7} \mathrm{i} r 0^{7} c 10\right. \\
& +1.292409115 \times 10^{16} \mathrm{i} \ln (r 0) c 7 r 0^{4}-3.000000000 \times 10^{9} c 10 r 0^{6}-6.692070654 \times 10^{17} c 7 r 0^{5} \\
& -1.440000000 \times 10^{11} r 0^{5} c 9-1000 \mathrm{i} c 7 r 0^{8}+4.896000000 \times 10^{9} \mathrm{i} r 0^{4} c 6-3.227234492 \times 10^{19} \mathrm{i} c 7 r 0^{2} \\
& +2.084590843 \times 10^{16} c 7 r 0^{7}-4.896000000 \times 10^{9} \mathrm{i} c 9 r 0^{6}+7.215316909 \times 10^{17} \ln (r 0) c 7 r 0^{5} \\
& +1.044710385 \times 10^{19} \mathrm{i} r 0^{4} c 7-1000 r 0^{8} c 7+1.884955592 \times 10^{11} r 0^{8} \rho \\
& -3.600000000 \times 10^{10} r 0^{7} c 9+8.16000000 \times 10^{8} \mathrm{i} r 0^{7} c 6-3.418052808 \times 10^{9} \mathrm{i} r 0^{7} \rho \\
& -6.836105613 \times 10^{9} \mathrm{i} r 0^{8} \rho+4.488000000 \times 10^{9} \mathrm{i} r 0^{6} c 6+3.015928948 \times 10^{11} r 0^{7} \rho \\
& +7.179615196 \times 10^{17} c 7 r 0^{4}+1.098242223 \times 10^{19} c 7 r 0^{3}+20000 \mathrm{i} r 0^{9} c 7+2.72000000 \times 10^{8} \mathrm{i} r 0^{6} c 10 \\
& +1.806026323 \times 10^{17} \ln (r 0) c 7 r 0^{6}-2.163898247 \times 10^{17} \mathrm{i} c 7 r 0^{6}+8.160000000 \times 10^{9} \mathrm{i} r 0^{5} c 6 \\
& +7.212387448 \times 10^{17} \ln (r 0) c 7 r 0^{4}+8.346481884 \times 10^{16} c 7 r 0^{6}-1.507964474 \times 10^{11} r 0^{6} \rho \\
& +2.111184000 \times 10^{19} c 7-1.200000000 \times 10^{10} c 10 r 0^{5}+1.382918067 \times 10^{18} \mathrm{i} c 7 r 0^{5} \\
& -1.311705542 \times 10^{16} \mathrm{i} r 0^{6} \ln (r 0) c 7-4.896000000 \times 10^{9} \mathrm{i} c 9 r 0^{5}-4.087019553 \times 10^{15} \mathrm{i} r 0^{7} \ln (r 0) c 7 \\
& +1.741939934 \times 10^{19} c 7 r 0+7.323651653 \times 10^{13} r 0^{7} \ln (r 0) c 7-6.491754249 \times 10^{20} \mathrm{i} c 7 r 0 \\
& -1.440000000 \times 10^{11} r 0^{6} c 9-4.113828618 \times 10^{16} \mathrm{i} c 7 r 0^{7}-2.563539606 \times 10^{9} \mathrm{i} r 0^{9} p \\
& +1.650271349 \times 10^{19} c 7 r 0^{2}-3.42398706 \times 10^{15} \mathrm{i} r 0^{5} \ln (r 0) c 7-8.031498194 \times 10^{20} \mathrm{i} c 7 \\
& \left.-1.200000000 \times 10^{10} c 10 r 0^{4}\right) /\left(r 0^{7}(r 0+2)^{2}\right),
\end{aligned}
$$

$R_{u u+}\left(r_{0}\right)=1 / 2\left(\left(r 0^{2}-2 r 0\right)\left(-915.9586340 \mathrm{i} c 2 / r 0^{3}+0.8160000000 \mathrm{i} c 4-0.6052309472 c 2\right.\right.$

$+6745.674492 c 2 / r 0^{4}+80948.09432 c 2 / r 0^{5}+7518.667272 \mathrm{i} c 2 / r 0^{4}+90224.00728 \mathrm{i} c 2 / r 0^{5}$

$\left.+1.503112547 \mathrm{i} c 2-69.78185262 \mathrm{i} c 2 / r 0^{2}-2733.489212 c 2 / r 0^{3}+12 c 1 / r 0^{3}+36.07819002 c 2 / r 0^{2}\right)$

$-7518.667272 \mathrm{i} c 2 / r 0^{2}-53.40273063 \mathrm{i} c 2 r 0+0.1360 \mathrm{i} r 0\left(1253.111212 \mathrm{i} c 2 / r 0^{2}-457.9793170 \mathrm{i} c 2 / r 0\right.$

$+7518.667272 \mathrm{i} c 2 / r 0^{3}-0.3026154736 c 2 r 0^{2}+22.10459629 c 2 r 0+1124.279082 c 2 / r 0^{2}$

$+6745.674528 c 2 / r 0^{3}+12 r 0 c 4+8.900455105 \mathrm{i} c 2 r 0+69.78185262 \mathrm{i} \ln (r 0) c 2+0.7515562734 \mathrm{i} c 2 r 0^{2}$

$\left.+0.4080000000 \mathrm{i} r 0^{2} c 4-1366.744606 c 2 / r 0+6 c 1 / r 0-36.07819002 \ln (r 0) c 2+c 5\right)$

$+1.815692842 c 2 r 0^{2}-132.6275777 c 2 r 0-6745.674492 c 2 / r 0^{2}-40474.04717 c 2 / r 0^{3}-72 r 0 c 4$

$+2747.875902 \mathrm{i} c 2 / r 0-45112.00363 \mathrm{i} c 2 / r 0^{3}-418.6911157 \mathrm{iln}(r 0) c 2-4.509337640 \mathrm{i} c 2 r 0^{2}$

$+8200.467636 c 2 / r 0-36 c 1 / r 0+216.4691401 \ln (r 0) c 2-6 c 5$

$+12 r 0\left(0.5043591226 \times 10^{-1} c 2-2.568292000 \times 10^{-11} c 2 / r 0-9.800840000 \times 10^{-11} \mathrm{i} c 2 / r 0\right.$

$-50.69289058 c 2 / r 0^{2}-0.680 \times 10^{-1} \mathrm{i} c 4+201.0479721 \mathrm{i} c 2 / r 0^{3}-0.1252593789 \mathrm{i} c 2$

$-6745.674528 c 2 / r 0^{5}+228.5542745 c 2 / r 0^{3}+1687.678839 \mathrm{i} c 2 / r 0^{4}-38.40352765 \mathrm{i} c 2 / r 0^{2}$

$\left.-7518.667272 \mathrm{i} c 2 / r 0^{5}+2 c 1 / r 0^{2}+2 c 1 / r 0^{3}+2614.546974 c 2 / r 0^{4}\right)$

$+0.8160 \mathrm{ir} 0^{3}\left(0.5043591226 \times 10^{-1} c 2-2.568292000 \times 10^{-11} c 2 / r 0-9.800840000 \times 10^{-11} \mathrm{i} c 2 / r 0\right.$

$-50.69289058 c 2 / r 0^{2}-0.680 \times 10^{-1} \mathrm{i} c 4+201.0479721 \mathrm{i} c 2 / r 0^{3}-0.1252593789 \mathrm{i} c 2$

$-6745.674528 c 2 / r 0^{5}+228.5542745 c 2 / r 0^{3}+1687.678839 \mathrm{i} c 2 / r 0^{4}-38.40352765 \mathrm{i} c 2 / r 0^{2}$

$\left.\left.-7518.667272 \mathrm{i} c 2 / r 0^{5}+2 c 1 / r 0^{2}+2 c 1 / r 0^{3}+2614.546974 c 2 / r 0^{4}\right)-2.448000000 \mathrm{i} r 0^{2} c 4\right) / r 0^{3}$, 


$$
\begin{aligned}
& R_{u r-}\left(r_{0}\right)=1.111111111 \times 10^{-9}\left(-1.272774256 \times 10^{19} \mathrm{i} c 7 r 0^{2}+1.314989772 \times 10^{20} \mathrm{i} c 7 r 0\right. \\
& -1.589998513 \times 10^{18} \mathrm{i} c 7 r 0^{3}+76 \mathrm{i} c 7 r 0^{6}+3.011811822 \times 10^{20} \mathrm{i} c 7+4.846534181 \times 10^{14} \mathrm{i} c 7 r 0^{4} \\
& +40952 \mathrm{i} r 0^{5} c 7-7.916940003 \times 10^{18} c 7+2.280721975 \times 10^{17} c 7 r 0^{2}-3.616361705 \times 10^{18} c 7 r 0 \\
& +8.260193057 \times 10^{16} c 7 r 0^{3}-3.02160 \times 10^{5} c 7 r 0^{5}-1.51080 \times 10^{5} c 7 r 0^{6} \\
& \left.+3.392920066 \times 10^{10} r 0^{6} \rho+2.704645291 \times 10^{16} c 7 r 0^{4}\right) /\left(r 0^{6}(r 0+2)\right) \text {, } \\
& R_{u r+}\left(r_{0}\right)=1 / 2\left(r 0 \left(-915.9586340 \mathrm{i} c 2 / r 0^{3}+0.8160000000 \mathrm{i} c 4-0.6052309472 c 2+6745.674492 c 2 / r 0^{4}\right.\right. \\
& +80948.09432 c 2 / r 0^{5}+7518.667272 \mathrm{i} c 2 / r 0^{4}+90224.00728 \mathrm{i} c 2 / r 0^{5}+1.503112547 \mathrm{i} c 2 \\
& \left.-69.78185262 \mathrm{i} c 2 / r 0^{2}-2733.489212 c 2 / r 0^{3}+12 c 1 / r 0^{3}+36.07819002 c 2 / r 0^{2}\right) \\
& +6 r 0^{2}\left(2.568292000 \times 10^{-11} c 2 / r 0^{2}+9.800840000 \times 10^{-11} \mathrm{i} c 2 / r 0^{2}+101.3857812 c 2 / r 0^{3}\right. \\
& -603.1439163 \mathrm{i} c 2 / r 0^{4}+33728.37264 c 2 / r 0^{6}-685.6628235 c 2 / r 0^{4}-6750.715356 \mathrm{i} c 2 / r 0^{5} \\
& \left.+76.80705530 \mathrm{i} c 2 / r 0^{3}+37593.33636 \mathrm{i} c 2 / r 0^{6}-4 c 1 / r 0^{3}-6 c 1 / r 0^{4}-10458.18790 c 2 / r 0^{5}\right) \\
& +12 r 0\left(0.5043591226 \times 10^{-1} c 2-2.568292000 \times 10^{-11} c 2 / r 0-9.800840000 \times 10^{-11} \mathrm{i} c 2 / r 0\right. \\
& -50.69289058 c 2 / r 0^{2}-0.680 \times 10^{-1} \mathrm{i} c 4+201.0479721 \mathrm{i} c 2 / r 0^{3}-0.1252593789 \mathrm{i} c 2 \\
& -6745.674528 c 2 / r 0^{5}+228.5542745 c 2 / r 0^{3}+1687.678839 \mathrm{i} c 2 / r 0^{4}-38.40352765 \mathrm{i} c 2 / r 0^{2} \\
& \left.\left.-7518.667272 \mathrm{i} c 2 / r 0^{5}+2 c 1 / r 0^{2}+2 c 1 / r 0^{3}+2614.546974 c 2 / r 0^{4}\right)\right) / r 0^{2}, \\
& q^{A} R_{u A-}\left(r_{0}\right)=1.000000000 \times 10^{-10}\left(16000 \mathrm{i} c 7 r 0^{8}-1.000000 \times 10^{6} r 0^{8} c 7+6.95123928 \times 10^{18} c 7 r 0^{4}\right. \\
& +4.60818516 \times 10^{20} \mathrm{i} c 7 r 0+6.811699261 \times 10^{15} \mathrm{i} c 7 r 0^{7}+1.202064574 \times 10^{18} \ln (r 0) c 7 r 0^{4} \\
& +1.140199529 \times 10^{19} \mathrm{i} c 7 r 0^{5}-7.391764651 \times 10^{20} \mathrm{i} c 7 r 0^{3}-1.220608500 \times 10^{14} c 7 r 0^{7} \\
& +4.461943440 \times 10^{21} \mathrm{i} c 7+300 r 0^{7} p+5.385037980 \times 10^{15} \mathrm{iln}(r 0) c 7 r 0^{6} \\
& -2.000000000 \times 10^{10} c 10 r 0^{4}+2.154015192 \times 10^{16} \mathrm{iln}(r 0) c 7 r 0^{5}-2.671516126 \times 10^{21} \mathrm{i} c 7 r 0^{2} \\
& +2.040000000 \times 10^{9} \mathrm{i} r 0^{6} c 6+3.005161436 \times 10^{17} \ln (r 0) c 7 r 0^{6}+8.160000000 \times 10^{9} \mathrm{ir} 0^{4} c 6 \\
& -2.000000000 \times 10^{10} c 10 r 0^{5}-5.000000000 \times 10^{9} c 10 r 0^{6}+1.202064574 \times 10^{18} \ln (r 0) c 7 r 0^{5} \\
& -3.817318694 \times 10^{18} c 7 r 0^{5}-6.543021412 \times 10^{16} \mathrm{i} r 0^{6} c 7+8.809405089 \times 10^{19} c 7 r 0^{3} \\
& +1.455381063 \times 10^{20} c 7 r 0^{2}+2.154015192 \times 10^{16} \mathrm{iln}(r 0) c 7 r 0^{4}-1.44797289 \times 10^{19} c 7 r 0 \\
& +8.160000000 \times 10^{9} \mathrm{ir} 0^{5} c 6+4.272566009 \times 10^{9} \mathrm{ir} 0^{9} \rho-3.509551026 \times 10^{17} c 7 r 0^{6} \\
& \left.+1.001418528 \times 10^{20} \mathrm{i} r 0^{4} c 7-1.172880000 \times 10^{20} c 7+8.545132018 \times 10^{9} \mathrm{i} r 0^{8} \rho\right) /\left(r 0^{6}(r 0+2)^{2}\right), \\
& q^{A} R_{u A+}\left(r_{0}\right)=1 / 2\left(-1253.111212 \mathrm{i} c 2 / r 0^{2}-c 5-1124.279082 c 2 / r 0^{2}+0.3026154736 c 2 r 0^{2}\right. \\
& -22.10459629 c 2 r 0-12 r 0 c 4+36.07819002 \ln (r 0) c 2-8 r 0^{2}\left(2.568292000 \times 10^{-11} c 2 / r 0^{2}\right. \\
& +9.800840000 \times 10^{-11} \mathrm{i} c 2 / r 0^{2}+101.3857812 c 2 / r 0^{3}-603.1439163 \mathrm{i} c 2 / r 0^{4} \\
& +33728.37264 c 2 / r 0^{6}-685.6628235 c 2 / r 0^{4}-6750.715356 \mathrm{i} c 2 / r 0^{5}+76.80705530 \mathrm{i} c 2 / r 0^{3} \\
& \left.+37593.33636 \mathrm{i} c 2 / r 0^{6}-4 c 1 / r 0^{3}-6 c 1 / r 0^{4}-10458.18790 c 2 / r 0^{5}\right) \\
& +r 0\left(457.9793170 \mathrm{i} c 2 / r 0^{2}+0.8160000000 \mathrm{i} r 0 c 4+8.900455105 \mathrm{i} c 2-0.6052309472 c 2 r 0\right. \\
& +22.10459629 c 2-2248.558164 c 2 / r 0^{3}-20237.02358 c 2 / r 0^{4}+12 c 4-2506.222424 \mathrm{i} c 2 / r 0^{3} \\
& -22556.00182 \mathrm{i} c 2 / r 0^{4}+1.503112547 \mathrm{i} c 2 r 0+69.78185262 \mathrm{i} c 2 / r 0 \\
& \left.+1366.744606 c 2 / r 0^{2}-6 c 1 / r 0^{2}-36.07819002 c 2 / r 0\right)
\end{aligned}
$$


$+4 r 0^{3}\left(2.568292000 \times 10^{-11} c 2 / r 0^{2}+9.800840000 \times 10^{-11} c 2 / r 0^{2}+101.3857812 c 2 / r 0^{3}\right.$ $-603.1439163 \mathrm{i} c 2 / r 0^{4}+33728.37264 c 2 / r 0^{6}-685.6628235 c 2 / r 0^{4}-6750.715356 \mathrm{i} c 2 / r 0^{5}$

$\left.+76.80705530 \mathrm{i} c 2 / r 0^{3}+37593.33636 \mathrm{i} c 2 / r 0^{6}-4 c 1 / r 0^{3}-6 c 1 / r 0^{4}-10458.18790 c 2 / r 0^{5}\right)$

$+r 0^{4}\left(-5.136584000 \times 10^{-11} c 2 / r 0^{3}-1.960168000 \times 10^{-10} \mathrm{i} c 2 / r 0^{3}-304.1573436 c 2 / r 0^{4}\right.$ $+2412.575665 \mathrm{i} c 2 / r 0^{5}-2.023702358 \times 10^{5} c 2 / r 0^{7}+2742.651294 c 2 / r 0^{5}+33753.57678 i c 2 / r 0^{6}$ $\left.-230.4211659 \mathrm{i} c 2 / r 0^{4}-2.255600182 \times 10^{5} \mathrm{i} c 2 / r 0^{7}+12 c 1 / r 0^{4}+24 c 1 / r 0^{5}+52290.93950 c 2 / r 0^{6}\right)$ $+2 r 0^{2}\left(0.5043591226 \times 10^{-1} c 2-2.568292000 \times 10^{-11} c 2 / r 0-9.800840000 \times 10^{-11} \mathrm{i} c 2 / r 0\right.$ $-50.69289058 c 2 / r 0^{2}-0.680 \times 10^{-1} \mathrm{i} c 4+201.0479721 \mathrm{i} c 2 / r 0^{3}-0.1252593789 \mathrm{i} c 2$

$-6745.674528 c 2 / r 0^{5}+228.5542745 c 2 / r 0^{3}+1687.678839 i c 2 / r 0^{4}-38.40352765 i c 2 / r 0^{2}$ $\left.-7518.667272 \mathrm{i} c 2 / r 0^{5}+2 c 1 / r 0^{2}+2 c 1 / r 0^{3}+2614.546974 c 2 / r 0^{4}\right)-8.900455105 \mathrm{i} c 2 r 0$ $-0.2720 \mathrm{i} r 0^{2}(c 4+c 1 / r 0+c 2(1+(13.04336905144130-1.31528646137769 \mathrm{i})(1 / r 0-1 / 6)$ $\left.\left.+(77.34402850-15.99899824 \mathrm{i})(1 / r 0-1 / 6)^{2}+(-187.3798480-208.8518687 \mathrm{i})(1 / r 0-1 / 6)^{3}\right)\right)$ $+457.9793170 \mathrm{i} c 2 / r 0-0.680 \times 10^{-1} \mathrm{i} r 0^{4}\left(2.568292000 \times 10^{-11} c 2 / r 0^{2}\right.$ $+9.800840000 \times 10^{-11} \mathrm{i} c 2 / r 0^{2}+101.3857812 c 2 / r 0^{3}-603.1439163 i c 2 / r 0^{4}$ $+33728.37264 c 2 / r 0^{6}-685.6628235 c 2 / r 0^{4}-6750.715356 \mathrm{i} c 2 / r 0^{5}+76.80705530 \mathrm{i} c 2 / r 0^{3}$ $\left.+37593.33636 \mathrm{i} c 2 / r 0^{6}-4 c 1 / r 0^{3}-6 c 1 / r 0^{4}-10458.18790 c 2 / r 0^{5}\right)$ $-7518.667272 \mathrm{i} c 2 / r 0^{3}-69.78185262 \mathrm{iln}(r 0) c 2-0.7515562734 \mathrm{i} c 2 r 0^{2}$ $-2 r 0^{3}\left(-5.136584000 \times 10^{-11} c 2 / r 0^{3}-1.960168000 \times 10^{-10} \mathrm{i} c 2 / r 0^{3}-304.1573436 c 2 / r 0^{4}\right.$ (65) $+2412.575665 \mathrm{i} c 2 / r 0^{5}-2.023702358 \times 10^{5} c 2 / r 0^{7}+2742.651294 c 2 / r 0^{5}+33753.57678 \mathrm{i} c 2 / r 0^{6}$ $\left.-230.4211659 \mathrm{i} c 2 / r 0^{4}-2.255600182 \times 10^{5} \mathrm{i} c 2 / r 0^{7}+12 c 1 / r 0^{4}+24 c 1 / r 0^{5}+52290.93950 c 2 / r 0^{6}\right)$ $\left.-0.4080000000 \mathrm{i} r 0^{2} c 4-6745.674528 c 2 / r 0^{3}+1366.744606 c 2 / r 0-6 c 1 / r 0\right) / r 0^{2}$,

\section{The Bondi Metric Variables Computed at $\boldsymbol{r}_{0}$}

$$
\begin{aligned}
U_{+}\left(r_{0}\right)= & 0.5043591226 \times 10^{-1} c 2-2.568292000 \times 10^{-11} c 2 / r 0-9.800840000 \times 10^{-11} \mathrm{i} c 2 / r 0 \\
& -50.69289058 c 2 / r 0^{2}-0.680 \times 10^{-1} \mathrm{i} c 4+201.0479721 \mathrm{i} c 2 / r 0^{3}-0.1252593789 \mathrm{i} c 2 \\
& -6745.674528 c 2 / r 0^{5}+228.5542745 c 2 / r 0^{3}+1687.678839 \mathrm{i} c 2 / r 0^{4}-38.40352765 \mathrm{i} c 2 / r 0^{2} \\
& -7518.667272 \mathrm{i} c 2 / r 0^{5}+2 c 1 / r 0^{2}+2 c 1 / r 0^{3}+2614.546974 c 2 / r 0^{4}, \\
U_{-}\left(r_{0}\right)= & \left(4.887000002 \times 10^{7}-1.859143100 \times 10^{9} \mathrm{i}\right) c 7 / r 0^{5} \\
& +4 c 7\left(2.865551000 \times 10^{6}-1.523351300 \times 10^{7} \mathrm{i}\right. \\
& \left.+\left(4.887000002 \times 10^{7}-1.859143100 \times 10^{9} \mathrm{i}\right)(1 / r 0-1 / 6)\right) / r 0^{4} \\
& +\left(-2.443500001 \times 10^{7}+9.295715500 \times 10^{8} \mathrm{i}\right) c 7 / r 0^{4} \\
& +\left(2 \left(c 6+c 7\left(29144-2.280672000 \times 10^{5} \mathrm{i}\right.\right.\right. \\
& +\left(2.865551000 \times 10^{6}-1.523351300 \times 10^{7} \mathrm{i}\right)(1 / r 0-1 / 6) \\
& \left.\left.\left.+\left(2.443500001 \times 10^{7}-9.295715502 \times 10^{8} \mathrm{i}\right)(1 / r 0-1 / 6)^{2}\right)\right)\right) / r 0^{3}
\end{aligned}
$$




$$
\begin{aligned}
& -c 7\left(2.865551000 \times 10^{6}-1.523351300 \times 10^{7} \mathrm{i}\right. \\
+ & \left.\left(4.887000002 \times 10^{7}-1.859143100 \times 10^{9} \mathrm{i}\right)(1 / r 0-1 / 6)\right) / r 0^{3} \\
& -\left(0.680 \times 10^{-1} \mathrm{i}\right) c 7\left(2.865551000 \times 10^{6}-1.523351300 \times 10^{7} \mathrm{i}\right. \\
+ & \left.\left(4.887000002 \times 10^{7}-1.859143100 \times 10^{9} \mathrm{i}\right)(1 / r 0-1 / 6)\right) / r 0^{2} \\
+ & \left(2 \left(c 6+c 7\left(29144-2.280672000 \times 10^{5} \mathrm{i}\right.\right.\right. \\
+ & \left(2.865551000 \times 10^{6}-1.523351300 \times 10^{7} \mathrm{i}\right)(1 / r 0-1 / 6) \\
+ & \left.\left.\left.\left(2.443500001 \times 10^{7}-9.295715502 \times 10^{8} \mathrm{i}\right)(1 / r 0-1 / 6)^{2}\right)\right)\right) / r 0^{2} \\
+ & \left(0.680 \times 10^{-1} \mathrm{i}\right)\left(c 6+c 7\left(29144-2.280672000 \times 10^{5} \mathrm{i}\right.\right. \\
+ & \left(2.865551000 \times 10^{6}-1.523351300 \times 10^{7} \mathrm{i}\right)(1 / r 0-1 / 6) \\
+ & \left.\left.\left(2.443500001 \times 10^{7}-9.295715502 \times 10^{8} \mathrm{i}\right)(1 / r 0-1 / 6)^{2}\right)\right) / r 0 \\
& -\left(0.680 \times 10^{-1} \mathrm{i}\right)(c 9+c 6 / r 0+c 7(-2518.141205+699.71985 \mathrm{i} \\
& +\left(29144-2.280672000 \times 10^{5} \mathrm{i}\right)(1 / r 0-1 / 6) \\
& +\left(1.432775500 \times 10^{6}-7.616756500 \times 10^{6} \mathrm{i}\right)(1 / r 0-1 / 6)^{2} \\
& \left.\left.+\left(8.145000002 \times 10^{6}-3.098571834 \times 10^{8} \mathrm{i}\right)(1 / r 0-1 / 6)^{3}\right)\right) \\
& +(0.4080000000 \mathrm{i}) r 0^{2} c 4-1366.744606 c 2 / r 0+6 c 1 / r 0-36.07819002 \ln (r 0) c 2+c 5 \\
\omega_{+}\left(r_{0}\right)= & (1253.111212 \mathrm{i}) c 2 / r 0^{2}-(457.9793170 \mathrm{i}) c 2 / r 0+(7518.667272 \mathrm{i}) c 2 / r 0^{3} \\
& -0.3026154736 c 2 r 0^{2}+22.10459629 c 2 r 0+1124.279082 c 2 / r 0^{2}+6745.674528 c 2 / r 0^{3} \\
+ & 12 r 0 c 4+(8.900455105 \mathrm{i}) c 2 r 0+(69.78185262 \mathrm{i}) \ln (r 0) c 2+(0.7515562734 \mathrm{i}) c 2 r 0^{2} \\
&
\end{aligned}
$$

and

$$
\begin{aligned}
\omega_{-}\left(r_{0}\right)= & -(2156.071282 \mathrm{i}) c 7 r 0^{2}-50.26548247 r 0^{2} \rho /(1+2 / r 0)+6 c 6 / r 0-5.147566058 \times 10^{5} c 7 r 0^{2} \\
& -63413.86124 c 7 r 0-2.932200001 \times 10^{8} c 7 / r 0^{3}-4.886999995 \times 10^{7} c 7 / r 0^{2} \\
& -2.356233015 \times 10^{8} c 7 / r 0-6.010322872 \times 10^{7} \ln (r 0) c 7+12 r 0 c 9+\left(1.513990017 \times 10^{7} \mathrm{i}\right) c 7 r 0 \\
& -\left(1.031580813 \times 10^{9} \mathrm{i}\right) c 7 / r 0+\left(1.859143099 \times 10^{9} \mathrm{i}\right) c 7 / r 0^{2}+\left(1.115485860 \times 10^{10} \mathrm{i}\right) c 7 / r 0^{3} \\
& +(0.4080000000 \mathrm{i}) r 0^{2} c 9-\left(1.077007596 \times 10^{6} \mathrm{i}\right) \ln (r 0) c 7+c 10,
\end{aligned}
$$

\section{The Computed Constants}

$$
\begin{aligned}
c 5= & -5.925925926 \times 10^{-21}\left(2.257876807 \times 10^{25} \mathrm{i} c 2 r 0+1.177568763 \times 10^{22} \mathrm{i} \ln (r 0) c 2 r 0^{4}\right. \\
& -2.537550206 \times 10^{25} \mathrm{i} c 2-1.179321011 \times 10^{22} \mathrm{i} c 2 r 0^{4}-6.885000000 \times 10^{19} \mathrm{i} r 0^{4} c 1 \\
& -2.276665152 \times 10^{25} c 2-1.379990759 \times 10^{20} \mathrm{i} c 2 r 0^{5}+8.836025110 \times 10^{9} c 2 r 0^{6} \\
& -6.088194566 \times 10^{21} \ln (r 0) c 2 r 0^{4}-3.251077700 \times 10^{23} \mathrm{i} c 2 r 0^{3}-6.873541974 \times 10^{23} \mathrm{i} c 2 r 0^{2} \\
& -2.669155863 \times 10^{20} c 2 r 0^{5}+8.950474631 \times 10^{21} c 2 r 0^{4}-4.171449115 \times 10^{23} c 2 r 0^{3} \\
& \left.+3.018766507 \times 10^{9} \mathrm{i} c 2 r 0^{6}-2.509446061 \times 10^{24} c 2 r 0^{2}+2.174295963 \times 10^{25} c 2 r 0\right) / r 0^{4},
\end{aligned}
$$




$$
\begin{aligned}
& c 7=\left(-1.683000000 \times 10^{14}+1.416666667 \times 10^{25} \mathrm{i}\right) c 6 /\left(-3.518394608 \times 10^{32}\right. \\
& \left.+9.145010221 \times 10^{31} \mathrm{i}-1.463828647 \times 10^{23} \ln (r 0)+\left(2.060201741 \times 10^{22} \mathrm{i}\right) \ln (r 0)\right), \\
& c 9=-1.111111111 \times 10^{-11}\left(1.157988867 \times 10^{17} r 0^{8} c 7-3.769911183 \times 10^{14} r 0^{7} \rho+5.22000 \times 10^{5} r 0^{9} c 7\right. \\
& -8.250022346 \times 10^{21} c 7 r 0^{4}+1.884955592 \times 10^{14} r 0^{6} \rho-2.356194490 \times 10^{14} r 0^{8} \rho \\
& -5.423600000 \times 10^{11} \ln (r 0) c 7 r 0^{5}+9.635910263 \times 10^{22} c 7 r 0^{2}-1.737134306 \times 10^{20} c 7 r 0^{6} \\
& +5.0000000 \times 10^{7} \mathrm{ir} 0^{7} \ln (r 0) c 7+6.926736238 \times 10^{21} c 7 r 0^{3}-8.000000 \times 10^{6} \mathrm{ir} 0^{9} c 7 \\
& -3.240686912 \times 10^{20} c 7 r 0^{5}-1.963050508 \times 10^{24} \mathrm{i} c 7 r 0^{2}-2.726124482 \times 10^{19} c 7 r 0^{7} \\
& -2.500000 \times 10^{6} r 0^{7} \ln (r 0) c 7+1.159077064 \times 10^{24} \mathrm{i} c 7 r 0-1.143558000 \times 10^{23} c 7 \\
& +2.863090381 \times 10^{20} \mathrm{i} r 0^{6} c 7+2.075034463 \times 10^{15} \mathrm{i} c 7 r 0^{8}+1.452672443 \times 10^{11} r 0^{9} \rho \\
& +2.000000000 \times 10^{9} \mathrm{i} \ln (r 0) c 7 r 0^{6}-6.319234788 \times 10^{23} \mathrm{i} c 7 r 0^{3}+1.495398102 \times 10^{13} \mathrm{i} r 0^{8} \rho \\
& +6.249786891 \times 10^{19} \mathrm{i} c 7 r 0^{7}+4.350394854 \times 10^{24} \mathrm{i} c 7-5.000000000 \times 10^{11} \ln (r 0) c 7 r 0^{4} \\
& +4.321899263 \times 10^{22} c 7 r 0+6.408849014 \times 10^{12} \mathrm{ir} 0^{9} \rho-9.236000000 \times 10^{10} \ln (r 0) c 7 r 0^{6} \\
& +6.704677816 \times 10^{21} \mathrm{i} c 7 r 0^{5}-4.080000000 \times 10^{12} \mathrm{i} r 0^{6} c 6-1.020000000 \times 10^{12} \mathrm{i} r 0^{7} c 6 \\
& -4.080000000 \times 10^{12} \mathrm{i} r 0^{5} c 6+6.054991092 \times 10^{22} \mathrm{i} r 0^{4} c 7+1.387200000 \times 10^{11} r 0^{5} c 6 \\
& +3.468000000 \times 10^{10} r 0^{7} c 6+7.000000000 \times 10^{9} \mathrm{i} \ln (r 0) c 7 r 0^{5}+1.387200000 \times 10^{11} r 0^{6} c 6 \\
& +2.500000000 \times 10^{9} \mathrm{i} \ln (r 0) c 7 r 0^{4}+4.272566010 \times 10^{12} \mathrm{i} r 0^{7} \rho \\
& \left.+7.263362215 \times 10^{10} r 0^{10} \rho\right) /\left(\left(17 \mathrm{i} r 0^{2}+500 r 0^{2}+68 \mathrm{i} r 0+2000 r 0+2000+68 \mathrm{i}\right) r 0^{5}\right),
\end{aligned}
$$

and

$$
\begin{aligned}
c 10= & 2.000000000 \times 10^{-10}\left(-3.509551026 \times 10^{17} c 7 r 0^{6}+1.202064574 \times 10^{18} \ln (r 0) c 7 r 0^{5}\right. \\
& +8.809405089 \times 10^{19} c 7 r 0^{3}-1.447972890 \times 10^{19} c 7 r 0-3.817318694 \times 10^{18} c 7 r 0^{5} \\
& +1.455381063 \times 10^{20} c 7 r 0^{2}-1.220608500 \times 10^{14} c 7 r 0^{7}+1.140199529 \times 10^{19} \mathrm{i} c 7 r 0^{5} \\
& -1.172880000 \times 10^{20} c 7+2.040000000 \times 10^{9} \mathrm{i} r 0^{6} c 6+6.951239280 \times 10^{18} c 7 r 0^{4} \\
& +3.005161436 \times 10^{17} \ln (r 0) c 7 r 0^{6}+4.608185160 \times 10^{20} \mathrm{i} c 7 r 0+4.461943440 \times 10^{21} \mathrm{i} c 7 \\
& +2.154015192 \times 10^{16} \mathrm{i} \ln (r 0) c 7 r 0^{5}+8.545132018 \times 10^{9} \mathrm{i} r 0^{8} \rho \\
& +1.202064574 \times 10^{18} \ln (r 0) c 7 r 0^{4}+1.001418528 \times 10^{20} \mathrm{i} r 0^{4} c 7+16000 \mathrm{i} c 7 r 0^{8} \\
& +5.385037980 \times 10^{15} \mathrm{iln}(r 0) c 7 r 0^{6}+8.160000000 \times 10^{9} \mathrm{i} r 0^{4} c 6+6.811699261 \times 10^{15} \mathrm{i} c 7 r 0^{7} \\
& -7.391764651 \times 10^{20} \mathrm{i} c 7 r 0^{3}-2.671516126 \times 10^{21} \mathrm{i} c 7 r 0^{2}+2.154015192 \times 10^{16} \mathrm{iln}(r 0) c 7 r 0^{4} \\
& -1.000000 \times 10^{6} r 0^{8} c 7+4.272566009 \times 10^{9} \mathrm{i} r 0^{9} \rho-6.543021412 \times 10^{16} \mathrm{i} r 0^{6} c 7 \\
& \left.+8.160000000 \times 10^{9} \mathrm{i} r 0^{5} c 6+300 r 0^{7} \rho\right) /\left(r 0^{4}\left(4+4 r 0+r 0^{2}\right)\right), \\
c 1= & (-196.9738585+23.32983310 \mathrm{i}) \mathrm{m}, \\
c 2= & (-1.651630988-1.544652377 \mathrm{i}) \mathrm{m}, \\
c 6= & (-197.3622743-4.147929487 \mathrm{i}) \mathrm{m} .
\end{aligned}
$$

